\title{
A genuine pediatric parapharyngeal abscess a year after uneventful adenotonsillectomy
}

\author{
Giuseppe Brescia ${ }^{1}$, Barbara Pedruzzi ${ }^{1,2}$, Roberto Stramare ${ }^{3}$, Alfonso Luca Pendolino ${ }^{1}$, \\ Alessandro Martini ${ }^{1}$, Gino Marioni ${ }^{1}$ \\ ${ }^{1}$ Department of Neurosciences DNS, Otolaryngology Section, Padova University, Padova, ${ }^{2}$ ENT Unit, Monselice Hospital, \\ Monselice, ${ }^{3}$ Department of Medicine DIMED, Padova University, Padova, Italy. \\ E-mail: giuseppe.brescia@aopdv.eneto.it \\ Received: 12th December 2016, Revised: 13th February 2017, Accepted: 20th February 2017
}

SUMMARY: Brescia G, Pedruzzi B, Stramare R, Pendolino AL, Martini A, Marioni G. A genuine pediatric parapharyngeal abscess a year after uneventful adenotonsillectomy. Turk J Pediatr 2017; 59: 715-718.

It is unusual for a parapharyngeal abscess (PA) to occur late after an adenotonsillectomy. We report herein a 13-year-old female patient who developed a PA a year after an uneventful adenotonsillectomy.

The patient underwent 2 surgical explorations and was given intravenous antibiotic treatment. She was discharged in good general condition after spending 7 days in hospital.

The potential pathophysiological mechanisms behind PA formation late after tonsillectomy or adenotonsillectomy, and rational treatments for PA are critically discussed.

Key words: parapharyngeal abscess, adenotonsillectomy, deep neck infection, pediatric, rational treatment.

Before the widespread use of antibiotics, several studies had shown that most deep neck infections (70-80\%) stemmed from complicated pharyngo-tonsillar infections, but there is now ample evidence of a declining incidence of deep neck infections of pharyngotonsillar origin. ${ }^{1}$ On the other hand, despite worldwide improvements in dental care and oral hygiene, several authors have reported that significant proportions (41-49\%) of deep neck infections are caused by dental infections. ${ }^{1}$ Parapharyngeal abscesses (PAs) are relatively common, but those occurring after tonsillectomy and/or adenotonsillectomy are unusual. In 1989, Stankiewicz et al. ${ }^{2}$ wrote that there had hitherto been no reports in the American medical literature of PAs occurring in patients previously treated with tonsillectomy, and they presented four original cases. PAs are the result of a cellulitis progressing to an organized phlegmon and subsequently maturing into an abscess. In the parapharyngeal space, abscesses can lead to severe and potentially even fatal complications ${ }^{3}$ such as airway compromise, aspiration pneumonia, invasion of contiguous structures, jugular vein thrombosis, Lemierre's syndrome, Horner's syndrome, mediastinitis, carotid artery hemorrhage, and sepsis. ${ }^{4}$

The aim of the present case report is to describe a new case of PA occurring in a pediatric patient who had undergone uncomplicated adenotonsillectomy a year earlier. The potential pathophysiological mechanisms behind the occurrence of this PA are discussed in detail below.

\section{Case Report}

In April 2015, a 13-year-old female patient was assessed at our Otolaryngology Section due to fever, trismus, dysphagia, and severe left neck pain extending to the ipsilateral shoulder. Four days beforehand, she had been examined at another hospital for sore throat and fever, and oral antibiotics (clarithromycin) and anti-inflammatory agents (ibuprofen) had been instituted. The patient had undergone an uneventful adenotonsillectomy a year earlier for recurrent acute tonsillitis and obstructive sleep apnea syndrome. 
Our Ear Nose Throat (ENT) examination revealed a limited medialization of the left pharyngeal wall. There was no macroscopic evidence of any residual tonsillar tissue. Flexible endoscopy of the upper airways showed a left nasopharyngeal wall medialization descending towards the oropharynx. Vocal fold motility was normal and the airway was patent. A left neck swelling was found, with antalgic neck stiffness. The patient was admitted at our unit with a laboratory profile showing neutrophil leukocytosis (white blood cells [WBC] $17.57 \times 10.9 / \mathrm{L}$; neutrophils [N] 16.49 $\mathrm{x} 10 .{ }^{9} / \mathrm{L}$ ), C-reactive protein [CRP] $160 \mathrm{mg} / \mathrm{L}$ ), while the electrolyte profile was normal. The coagulation indexes showed a prothrombin time (PT) of $57 \%$ and an International Normalized Ratio (INR) of 1.2.

An urgent contrast-enhanced CT scan of head and neck was performed, revealing a large left parapharyngeal and retropharyngeal fluid collection extending from the nasopharynx to the oropharynx, with thickened, hyperdense walls. This collection came close to the skull base, and displaced the upper airway (Fig. 1. a,b,c). An urgent contrast-enhanced magnetic resonance imaging (MRI) could not be performed at admission because of a fixed orthodontic appliance. The patient underwent transoral incision and drainage of the PA under general anesthesia, removing approximately $9 \mathrm{ml}$ of purulent material. Microbiological cultures were negative for bacterial growth. After surgery, the patient was monitored for 48 hours at the pediatric postoperative intensive care unit. Intravenous ceftriaxone (1g twice a day) and metronidazole (500 mg three times a day) were administered. Although the CRP and WBC dropped significantly (CRP $53.2 \mathrm{mg} / \mathrm{L}$; WBC $10.15 \times 10.9^{9} \mathrm{~L}$, with $\mathrm{N} 8.03 \times 10.9^{9} / \mathrm{L}$ ) by the 3rd day after admission, the coagulation indexes were still altered (PT 50\%; INR 1.27), and a follow-up contrast-enhanced CT scan (on the 3rd day after admission) showed thickened vascularized tissue containing a

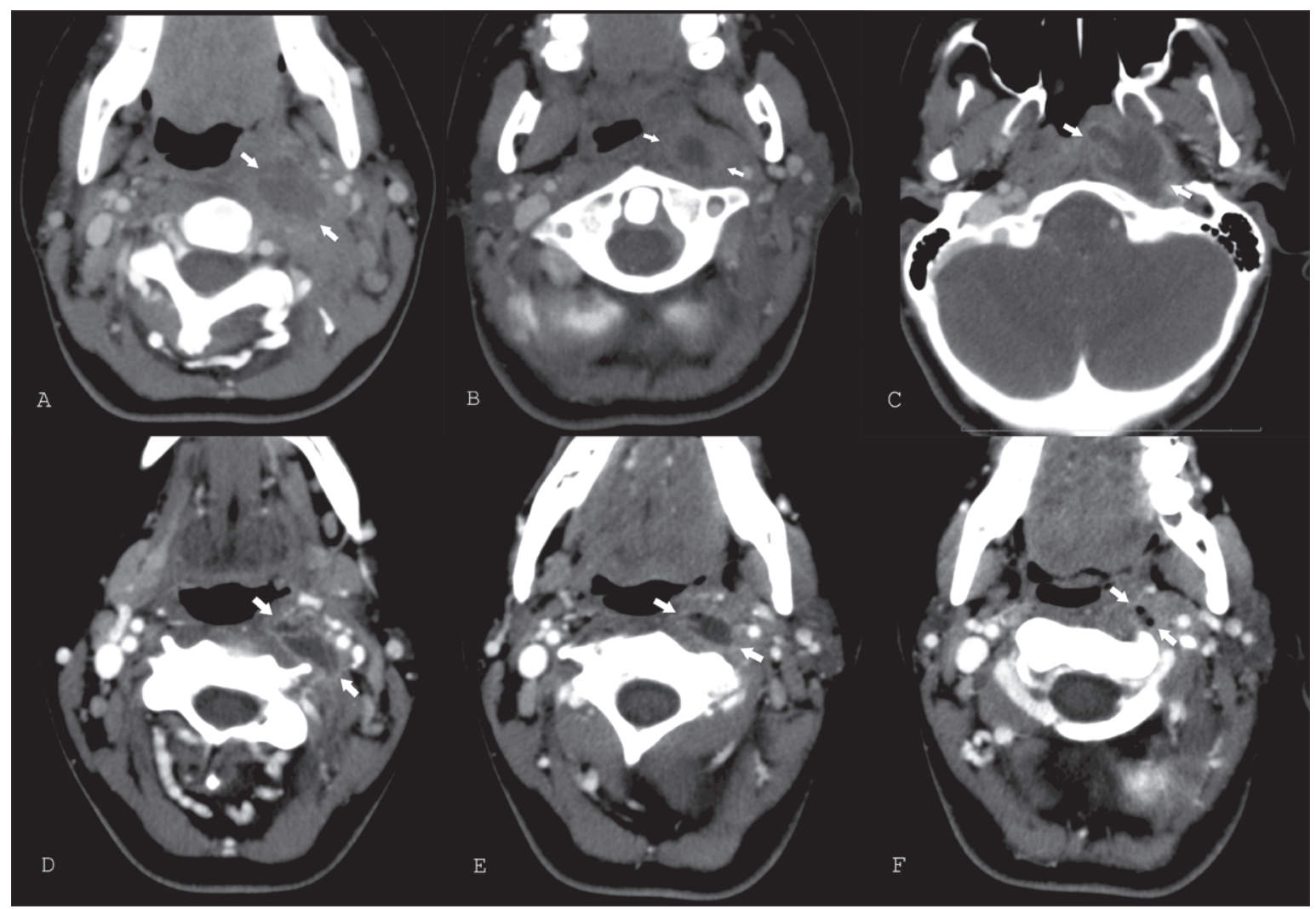

Fig. 1. a,b,c. large left parapharyngeal and retropharyngeal fluid collection extending from the nasopharynx to the oropharynx, with thickened, hyperdense walls (white arrows); the fluid collection comes close to the base of the skull and displaces the upper airway $(a, b)$. d. e. f. after surgery, contrast-enhanced CT shows persistence of the fluid collection (white arrows) with signs of the presence of gas bubbles (f). 
fluid collection surrounded by a hyperdense halo, with the appearance of gas bubbles, in the left para-nasopharyngeal region. The inflammatory process displaced contra-laterally and partially compressed the air column, and involved the carotid spaces, exerting pressure on the upper portion of the internal jugular vein (Fig. 1. d,e,f). Given this clinical and radiological evidence, drainage of the residual abscess was performed, removing approximately $4 \mathrm{ml}$ of dense, yellowish purulent material. Microbiological cultures were positive for Streptococcus mitis, Lactobacillus rhamnosus and Candida spp. The CRP, WBC, and neutrophil counts were normal on the 6th day after admission. Flexible endoscopy of the upper aerodigestive tract was normal and the patient was discharged on the 7 th day after admission. Further oral antibiotic treatment was prescribed (cefpodoxime $200 \mathrm{mg}$ twice a day, and metronidazole $250 \mathrm{mg}$ twice a day, for 6 days). A week after being discharged, the patient was reassessed in an out-patient setting. She showed no sign of infection or remnant tonsils. A contrast-enhanced CT scan of head and neck was scheduled after six months to rule out any presence of congenital anomalies as branchial cysts and fistulas. The parents refused to perform MRI or cone beam CT scan after discharge for reasons of cost.

Patient's parents signed a written permission for clinical case publication.

\section{Discussion}

It is unusual for a PA to occur after an uneventful adenotonsillectomy in childhood ${ }^{2}$. In our recent experience, among the last 38 patients treated for PAs, we found only one who had undergone adenotonsillectomy. On the other hand, in a series reported by Duval and Daniel ${ }^{4}, 25$ (13.9\%) of 180 children admitted for retro- and/or parapharyngeal infections at the Department of Otolaryngology in Montreal (Canada) had already undergone adenotonsillectomy (the authors considered both abscesses and phlegmons).

Reviewing the literature brings to light several possible explanations for PAs developing after an uncomplicated tonsillectomy. The most frequently mentioned is probably that PAs could develop from infected tonsillar capsule or tonsillar tissue remnants. ${ }^{5}$ Other possible causes of PA after uneventful tonsillectomy or adenotonsillectomy have been reported, including: (i) infections of branchial cysts and fistulas (occasionally the membrane between the second branchial pouch and cleft persists, giving rise to an internal branchial cyst or fistula) in continuity with the tonsillar cavity; (ii) ingestion of foreign bodies (fish bones or chicken bones); (iii) traumas (be they iatrogenic, caused by laryngoscopy, endotracheal intubation, feeding tube placement, etc., or otherwise, e.g. gunshot injury); and (iv) complications of dental infections. ${ }^{2,5,6}$ Considering children who have undergone tonsillectomy/adenotonsillectomy in particular, some authors speculated that PAs might form as a result of infections in the nose, ear or pharynx, involving the back of the tongue where Weber's glands are situated, and spreading to the lymph nodes of the parapharyngeal and retropharyngeal spaces, giving rise to an acute suppurative lymphadenitis. ${ }^{7,8}$ Such a mechanism could also relate to the abundance of lymphatic tissue in the parapharyngeal and retropharyngeal spaces in children, and/or to a momentary decline in local defenses.

General predisposing factors for the occurrence of PAs in patients who have undergone tonsillectomy/adenotonsillectomy should not be underestimated. It has been reported that adenoidectomy and tonsillectomy can lead to lower levels of local immunoglobulins, resulting in a weakening of the immune system, and thereby contributing to the spread of infections. ${ }^{4,9,10}$ Weaker local immune defenses could also be associated with certain disorders affecting the immune system (e.g. diabetes, malnutrition, recent EBV infection, HIV, or cancer), or with the use of immunosuppressive therapies (corticosteroids, chemotherapy), or personal habits (drug use, smoking).

Our patient had undergone an uneventful adenotonsillectomy a year before her PA developed. No disorders were diagnosed in our patient that might have impaired her immune system. In the present case, the origin of the PA could not be an adeno-tonsillar tissue infection, as quite frequently happens. Considering the clinical history of the patient and the ENT evaluation, we hypothesized two reasonable explanations for PA development. She had a fixed orthodontic appliance that 
might have been responsible for periodontal tissue/structure injuries. Dental manipulations have already been described as potentially leading to PA formation ${ }^{6}$. Repeated orthodontic procedures and injuries may well have caused recurrent asymptomatic oral infections relating to the development of a PA in the present case too. On the other hand, although it was very likely that the PA could occur regardless the previous adenotonsillectomy, our patient showed scarring of the tonsillar pillars after this surgical procedure, and Arciello et al. ${ }^{11}$ hypothesized that wound healing after tonsillectomy might create a partial fusion of the palatine pillars, predisposing to abscess pathogenesis.

In para-pharyngeal infections, surgical exploration and drainage may be mandatory already at presentation (particularly when contrast-enhanced imaging shows a sizable, encapsulated, hypodense and heterogeneous lesion, and/or potentially life-threatening complications such as descending mediastinitis or jugular vein thrombosis), or when clinical and laboratory findings indicate that a patient has failed to respond to parenteral antibiotics within $24-48 \mathrm{~h}^{12}$. In similar pediatric patients MRI should be definitely preferred; in particular, frequent CT scans in short time periods should be avoided in children except conditions of utmost seriousness. As in the reported case, multiple surgical explorations and drainage procedures may be needed before any significant improvement is seen in a patient's condition.

\section{Acknowledgements}

The authors thank Frances Coburn for correcting the English version of this paper.

\section{REFERENCES}

1. Favaretto N, Fasanaro E, Staffieri A, et al. Deep neck infections originating from the major salivary glands. Am J Otolaryngol. 2015; 36: 559-564.

2. Stankiewicz JA. Talland C. Peritonsillar like lateral oropharyngeal abscess after tonsillectomy. Arch Otolaryngol Head Neck Surg 1988; 114: 1181-1183.

3. Jin L, Zhang T. Deep neck infections: a retrospective study of 142 patients. B-ENT 2014; 10: 127-132.

4. Duval M, Daniel SJ. Retropharyngeal and parapharyngea abscesses or phlegmon in children. Is there an association with adenotonsillectomy? Int J Pediatr Otorhinolaryngol 2008; 72: 1765-1769.

5. Farmer SE, Khatwa MA, Zeitoun HM. Peritonsillar abscess after tonsillectomy: a review of the literature. Ann R Coll Surg Engl 2011; 93: 353-355.

6. Harkani A, Hassani R, Ziad T, et al. Retropharyngeal abscess in adults: five case reports and review of the literature. ScientificWorldJournal 2011; 11: 1623-1629.

7. Arora S, Sharma JK, Pippal SK, Yadav A, Najmi M Singhal D. Retropharyngeal abscess following a gun shot injury. Braz J Otorhinolaryngol 2009; 75: 909.

8. Marques PM, Spratley JE, Leal LM, Cardoso E, Santos M. Parapharyngeal abscess in children: five year retrospective study. Braz J Otorhinolaryngol 2009; 75: 826-830.

9. Cantani A, Bellioni P, Salvinelli F, Businco L. Serum immunoglobulins and secretory IgA deficiency in tonsillectomised children. Ann Allergy 1986; 57: 413 416.

10. Jeschke R, Stroder J. Continual observation of clinical and immunological parameters, in particular of salivary IgA, in tonsillectomized children. Acta Otorhinolaryngol 1980; 226: 73-84.

11. Arciello F, Crosetti E, Di Lisi D, Succo G. Parapharyngeal abscess two years after elective tonsillectomy. J Med Cases 2014; 5: 295-297.

12. Marioni G, Staffieri A, Parisi S, et al. Rational diagnostic and therapeutic management of deep neck infections: analysis of 233 consecutive cases. Ann Otol Rhinol Laryngol 2010; 119: 181-187. 